\title{
Overflow Controlled Declining Rate Filtration: A Conceptual Design for Increased Flexibility and Dynamism
}

\author{
Ababu T. Tiruneh"1, Tesfamariam Y. Debessai ${ }^{2}$, Gabriel C. Bwembya², Stanley J. Nkambule \\ ${ }^{1}$ Department of Environmental Health Science, University of Eswatini, Mbabane, Eswatini \\ ${ }^{2}$ Department of Chemistry, University of Eswatini, Mbabane, Eswatini \\ Email: ababute@gmail.com
}

How to cite this paper: Tiruneh, A.T., Debessai, T.Y., Bwembya, G.C. and Nkambule, S.J. (2019) Overflow Controlled Declining Rate Filtration: A Conceptual Design for Increased Flexibility and Dynamism. Journal of Water Resource and Protection, 11, 1245-1259.

https://doi.org/10.4236/jwarp.2019.1110072

Received: August 31, 2019

Accepted: October 8, 2019

Published: October 11, 2019

Copyright $\odot 2019$ by author(s) and Scientific Research Publishing Inc. This work is licensed under the Creative Commons Attribution International License (CC BY 4.0).

http://creativecommons.org/licenses/by/4.0/

\begin{abstract}
A declining rate filter operating through over flow control mode is proposed in this paper with a view to improving the overall performance of declining rate filters. Traditional declining rate filters have in-built self-adjustment of flow rates among the filters assisted with downstream orifice for flow control with limited flexibility for further adjustment. The proposed design allows the control of flow level and flow rates in individual filters, the water level in the over flow distributor tank and offers increased flexibility in responding to changes in filtration variables and optimising the overall performance of the filters. The design set up can also operate as constant rate filter through setting the water level in the distributor tank to the maximum.
\end{abstract}

\section{Keywords}

Water Treatment, Filtration, Declining Rate Filters, Variable Rate Filters, Constant Rate Filters, Filter Run

\section{Introduction}

Filtration is an important treatment process employed in modern water treatment plants and is traditionally considered as the last physical unit process in the chain of multiple barrier system existing in water treatment for solids removal. Carryover of coagulated solids from sedimentation is removed by filtration [1]. Although not the last barrier for the removal of pathogenic organisms, filtration also assists in the removal of Giardia Lamblia and Cryptosporidium [2] [3]. The remaining pathogens are expected to be eliminated by the last barrier system for pathogens removal, namely, disinfection [4]. Filters do also have the 
capability of removing iron and manganese as contact media facilitating co-adsorption [5]. Filters operating in rapid mode (rapid sand filters) are common in conventional installations because of their low space requirements and provision for self-cleaning using the system of backwashing. They continue as component units of conventional water treatment installations despite the emergence of additional superior treatment technologies such as micro filters and ultra-filters [6].

There are two commonly adopted modes of operation of rapid sand filters, namely constant flow rate and declining flow rate modes. Constant flow rate filters have uniform flow rate in all the filters throughout the stages of filter operation and operate in three possible modes [7]. In the downstream valve control mode of operation, the downstream valve is gradually opened while the water level in the filter remains constant. In the constant filter water level mode of operation, a water level rise in the filter in response to increase in head loss triggers a sensor that automatically adjusts the flow rate by opening the downstream valve further. Lastly, in the rising filter water level mode of operation, uniform flow splitting devices such as weirs are provided in which the flows are equally distributed among the filters while constant flow conditions in individual filters are maintained through proportional rise in water level in the filters as more head loss is developed during operation.

In rapid sand filters operating in the declining mode, the water level in all the filters stays the same and the filters operate with varying filtration rates. The water normally flows from the common channel and enters through each filter at a level that is set below the minimum water level [8]. This distribution of water to the filter to a common water level does not require any special uniform flow distribution device. The filter that is washed most recently carries higher flow rate whereas the dirtiest filter that has run the longest time carries the least flow [8]. This means that, the flow rate within each filter progressively decreases from the maximum (immediately following backwashing) to the minimum (when the filter is dirty and needs to be taken off for backwashing). This mode of operation facilitates longer filter run as filters with clogged conditions are allowed to operate at low flow rates which will prevent the development of higher head loss. Operation at low flow rate with associated low head loss development enables filters to continue operating for a longer period before they are taken out for cleaning.

\section{Declining Rate Filtration and Their Performance}

Declining rate filtration is a well-known filtration mode in which the water flow rate in an individual filter is allowed to drop over time. This mode of filtration acquired its name because of such decrease in flow rate with time. Operation of declining rate filters is different from the traditional constant flow rate filters in which the flow rate in a filter stays more or less constant throughout the run of filtration. By allowing the filtration rate to decrease with time, it is possible to 
achieve extended period of operation before a particular filter is taken out for back washing. As filters become lodged with deposits, their ability to accept flow and filter solids diminishes. Therefore, declining rate filter takes account of such limitation in performance and reduces flow rates in dirty filters so that they can continue operating with the same efficiency of filtration. The flow rejected by the dirty filters is distributed to the clean filters and because of this a minimum of four filters are required in order to run a filter system in declining mode enabling only moderate increase in filtration rate as result of sharing of the rejected excess flow during cleaning by the other three filters that are in operation. The minimum of four filters is also needed for another reason. It is possible to wash the dirtiest filter using the filtered water from the other filters. This arrangement of washing filters using effluent from the other filters avoids the need for more expensive backwash system [9]. The filtered water produced from the three filters that are in operation will provide enough volume of water for washing when the dirtiest filter is taken out for backwashing.

One advantage of declining rate filters is the absence of the need for special inflow splitting device or uniform flow distribution device for inlets to the different filters [10]. The filters are interconnected through a common inlet pipe that ensures that the water level is the same in each of the filters and flow to the filters enter below the minimum water level.

\subsection{Mode of Operation of Declining Rate Filters}

Declining rate filters operate with constant water level common to all the filters whereas the flow rate in each of the filters varies depending on the amount of solids accumulated in the filters. The water level rises during operation commonly for all the filters, the rise in water level is in response to the head loss build up among the different filters that are in different stages of operation [11]. The initial high flow rate through the most recently washed filter is restricted by providing a downstream orifice that responds with high loss in response to high flow rate tendency. Meanwhile, this same orifice located in the dirtiest filter has low head loss and would not inhibit the flow in this particular filter. Alternatively, head loss control can be provided through downstream valve with the attendant problem of constant operational attention and flow disturbance.

Declining rate filters require a minimum of four identical filters. The inflow to the filters should be located below the lowest water level so that all the filters operate at the same water level. The head loss through the pipe work is negligible [12]. The water level following backwashing of one of the filters immediately drops in all the filters, which results somehow in change of the flow regime in each of the filters depending on their stage of operation. The most recently washed filter now carries the highest flow rate whereas the rest of the other filters will have their flow rate reduced as result. Figure 1 shows the filtration and head loss change following backwashing of one of the filters. 


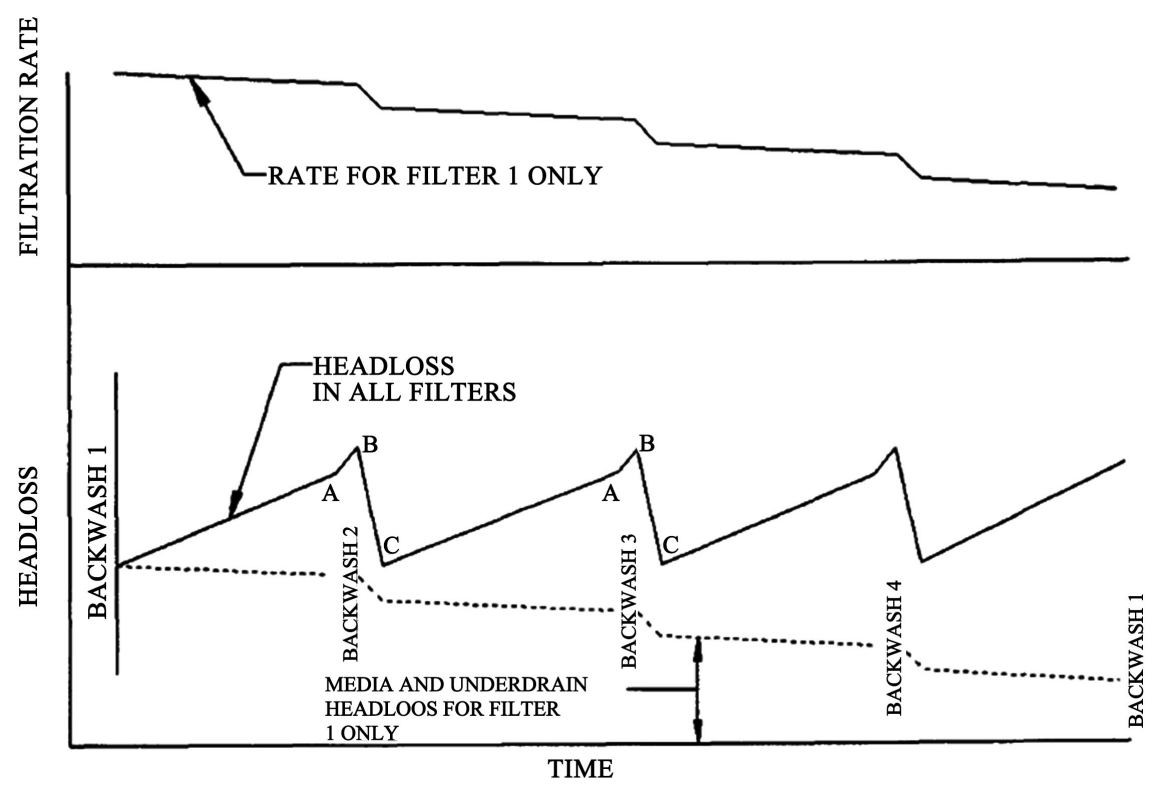

Figure 1. Filtration rate and head loss during one filter run in declining rate filters [14].

\subsection{Filter Flow Control in Declining Rate Filters}

Declining rate filters have two modes of flow control. In the first mode of flow control, the water level rises from the minimum following backwash of the dirtiest filter to the maximum when another filter need to be taken out for backwashing [13]. This mode of operation is easier as the flow rate is kept constant through rise in common water level in the filter. However, it requires additional wall to be built up to allow for rise in water level as the filter head loss increases. In the second mode of operation, the outlet weir level is lowered following increase in head loss whereas the water level in the filters remains more or less constant. This mode of operation is difficult requiring periodic adjustment in response to head loss build up.

Filter flow control through declining mode does not require any further adjustment than the static provisions in the form of downstream flow restricting orifices. On the other hand, compared to filters operated by downstream flow regulators, there is an economic advantage to be gained while at the same time maintaining comparable quality of filtered water [15]. The flow-restricting device is designed in such a way that the maximum hydraulic load (flow rate) in each of the filters does not exceed 1.5 times the average flow rate [8].

\subsection{Head Loss Development in Declining Rate Filters}

In declining rate filters, the initial head loss following backwashing of the filters is largely due to the flow-restricting orifice that is provided downstream for the purpose of limiting high flow rate that results in deterioration of water quality. This head loss is turbulent. Therefore, the head loss is proportional to the square of the flow rate. On the other hand as the filtration progresses towards the end of the filter run, the head loss will be largely due to solids build up in the filter, 
which is inherently a laminar head loss [11] [16] [17]. At this time, because of the low rate of filtration, the head loss in the flow-restricting orifice will be comparatively low. This mode of head loss transition from turbulent to laminar head loss has the advantage of lowering the hydraulic load as the filters become dirty as laminar flow vary only linearly with the flow rate.

When dual media filtration is used, such as a combination of coarse anthracite at the top and silica sand at the bottom, greater change in head loss occur in the anthracite media as a result of the proportionally greater amount of solids deposited on the coarser anthracite media. This observation may be similar to the ones observed in constant flow rate filters but can be a critical factor in the increase in turbidity at later stages of operation after the anthracite is clogged because of the fact that the sand may have limited solids storage capacity [18].

\subsection{Filtered Water Quality}

In terms of water quality, declining mode of operation has been observed to result in improved filtered water quality because of better distribution of solids achieved through reduced filtration rate. When the filtration rate is high, one part of the sand bed gets too much of the solids and gets clogged resulting in significant head loss before the remaining portion of the sand is able to deposit solids. This uneven distribution of solids results in loss of efficiency of performance and lowered filter runs [15] [19].

According to Marzec and Dobrowski [20], operation of rapid sand filters under different raw water turbidity did not materially alter the flow rates prevalent in the different filters as long as the water level following backwashing of one of the filters stays the same. This indicates that under stable operational conditions in terms of water level being the same following filter backwashes, variation of raw water quality does not seem to have significant effect on the distribution of flow rate among the different filters operating in declining mode. On the other hand, the same researchers indicated that the filtered water quality as well as the filter run times was significantly affected by variation in the raw water quality directed to the filters operating in declining mode. On the other hand, environmental variables such as seasonal changes in water temperature could not be regulated by the same self-controlled flow mechanism present in declining rate filters necessitating different and potentially tedious adjustment procedure. This is one of the drawbacks of filters operating in declining mode [21].

\subsection{Number of Filters Operating in Parallel}

When the number of filters operating in declining mode increases, there will be improvement in filtered water quality because of the improvement of filtered water turbidity as filtration progresses. In effect, the loss in the filtrate quality of the fresh filters will be compensated by the improved filtrate quality of the filters operating at low filtration rates. Larger plants may have their number of filters exceeding 30 favouring this marked improvement in filtrate quality [22]. 


\subsection{Comparison of Performances of Constant and Declining Rate Filters}

Constant flow rate filters often require frequent backwashing due to the limited filter run time because of rapid head loss development and deterioration of filtrate quality. Such frequent backwashing results in disruption of the filtration process as well as requiring increased volume of water required for backwashing. Declining rate filters reduce the frequency of disruption by allowing longer filter run while at the same time minimizing the amount of water needed for backwashing per a given time [23]. Past studies also demonstrated that, despite the initially high flow rate through backwashed filters, the filtered water turbidities were comparable with those of constant rate filters [19] [24].

There are, however, few-apparently contradictory-claims and counter claims cited in the literature regarding some of the advantages associated with the operation of declining rate filters. For example, it helps to look at the research conducted by the American Water Works Research Association [7]. The research study found little difference between the two modes of filtration in terms of flow disturbance, turbidity increase and overall cost. Both modes of operation were recommended with the only difference stated being ease of operation; the advantage lying with operation of declining rate filters.

On the other hand, Perera et al. [18] found out that declining rate operation produced, in general, better filter performance with improved filtered water turbidity and improved head loss conditions. The improved performance in terms of head loss is attributed to better redistribution of solid (solids penetration) that is achieved with declining mode of operation.

\subsection{Optimisation of Filter Performance}

In studies related to performance of declining rate filters, the total water flow flittered and the maximum allowable water level are considered constant (given). The variables considered in the optimisation process include: Filtration rate, medium size, inflow water quality, filter run, minimum water level, downstream head loss control, number of filters, etc. [13]

\subsection{Why Choose Declining Rate Filters?}

Declining rate filters offer an economical advantage of producing more water per filter run because of the privilege of extended period of operation of individual filters at declining filtration rates [7]. In addition, cleaning frequency is reduced in declining rate filters with less interruption in filter operations and less water used for backwashing [25]. Better-filtered water quality is produced for settled water after coagulation as well as coagulated water intended for direct filtration. Less hydraulic load is needed in the operation of declining rate filters [8]. On the other hand, in constant rate filters, extended time of backwashing and the resulting lost time of filtration need to be compensated for by provision of extra filter capacity so that the same desired flow-through can be maintained [26]. 


\subsection{Drawbacks with Design and Operation of Declining Rate Filters}

Declining rate filters have a number of disadvantages. Lack of operational flexibility is one disadvantage of declining rate filters although this too can be an advantage as operational intervention is minimised. When the flow rate changes, because of change in design conditions or when one of the filters has to be taken out of operation for maintenance purposes, the condition of operation changes and may be far from what is required to be optimum condition for satisfactory performance of the filters. There is no simple operational means to adjust to these new conditions without resorting to major modification in structural design of the filters.

Another disadvantage of declining rate filters is the fluctuation in flow rates in the periods during back washing, after backwashing and as the filter progresses towards its end of filter run. The sudden change in filtration rate is observed in all the filters immediately after a filter has been backwashed and been put back into operation [23]. Such rapid change in filtration coupled with the existence of residual solids in the recently washed filter can result in upset of the conditions of solids storage on the filter bed in general and deterioration of filtrate quality in particular. Such flow rate fluctuations give rise to different filter performance affecting the quality of the filtered water [27]. There is also a sharp water level rise when one of the filters is isolated for backwashing resulting in sudden change in flow rates among the remaining filters that are in operation.

The lowering of the common water level following backwashing is as stated a condition that has an effect on the distribution of flow rates among the different filters. On the positive side, flow rates in the dirtier filters are reduced, and the recently washed filter carries the excess flow discarded by the dirty filters. Such redistribution of flow facilitates operation of filters with longer filter runs and better distribution of solids deposit on the filter bed. On the other hand, the proportion of flow distribution among the different filters cannot be controlled and may change when the flow rate changes (because of increased demand or when a filter is taken out for maintenance) or when the raw water quality changes.

According to Murat et al. [28], a study of the impact of initial water quality and flow rate in declining rate filters revealed that the ratio between the maximum and minimum filtration rates among the different filters decreased when the flow rate in the system increases. Such reduction in filtration velocity ratio results in reduced efficiency of flow redistribution among the different filters, particularly reduction in the ability of the cleanest filter to share a larger proportion of the flow. The same ratio between maximum and minimum filtration rates increased when the coagulant dosage added to the raw water was reduced, indicating reduced efficiency of flow passage through the dirtier filters.

The provision of downstream flow restricting orifice is the only means available to restrict the flow rate fluctuation to desirable range. The absence of conve- 
nient operational mechanism to adjust to changing operational conditions is the inherent drawback of declining rate filters. Declining rate filters are, therefore, considered optimum in their performance only with respect to narrowly defined operational conditions. Development of self-controlled mechanisms to increase flexibility of operation of declining rate filters do not practically exist and are not reported in the literature.

\subsection{Addressing the Problems of Operation of Declining Rate Filtration}

Declining rate filters have a number of problems related mainly to their lack of operational flexibility and sudden fluctuation of flow rate in response to backwashing of the filters. There will be deterioration of water quality at high initial flow rate of the filters following backwashing as well as during backwashing operations. The sudden change in flow rate also has an effect on solids deposition in filter media and can potentially upset filter performance through dislodging of deposited solids. Declining rate filters can require major structural readjustment whenever the flow rate changes or the raw water quality changes or in response to changes in environmental variables such as seasonal temperature variation. This is not simple and routine task since structural adjustments such as changing the operating water level, surface area of filters and number of filters that are necessitated by change of environmental variables require major physical alterations to the original filter design.

Because of the same water level commonly prevailing in all the filter beds, the distribution of flow rates among the filters can be less than optimum whenever the design variables change such as the overall flow rate or the coagulant dosage. This is apparently lack of flexibility that is inherent in declining rate filters. The self-control adjustment of flow rate in response to head loss development through orifices in declining rate filters does not work for changes in environmental variables such as temperature difference that are routinely observed between different seasons since the orifice does not respond to changes in temperature [23]. There is, therefore, a need to consider an alternative setup such as this proposed overflow-controlled dynamic filter system to investigate the possibility of adaptation to changes in design variables without making major structural adjustment to the filter.

\section{The Concept of Overflow Controlled Declining Rate Filtration}

In order to overcome such inherent problems with the operation of declining rate filters and to provide a more dynamic operational condition for optimising the performance, a concept of overflow-controlled variable rate filtration is proposed in this paper. The idea of this alternative set up for filtration is based on the provision of separate water levels for each filter while the whole filter setup is interconnected through an overflow distributor tank. The proposed setup allows flexibility in controlling flow rate changes in individual filters so that wide fluc- 
tuations are minimised or the influence of flow rate changes in other filters such as the recently back-washed one has less negative impact on the operation of the other matured filters. Additional flexibility of level changes and valve setup are also included in this new conceptual design to provide more flexibility in response to changes in design conditions, situations that are often difficult to handle in the existing declining rate filter setups. However, filtration performance studies of new systems or improvement of existing systems always require pilot testing since analytical models that enable full design of filters on theoretical assumptions alone do not exist [29].

The concept of overflow-controlled declining rate filtration is here by proposed in this paper with the aim of addressing the operational setback of declining rate filters through the provision of a more flexible control in the operation of the filters. Such added control involves mechanisms such as: The provision of variable water level in each filter participating in the filter, provision for adjustment of water level in the overflow distributer tank, and flow restricting mechanisms such as orifices provided upstream of the filters to each of the individual filters operating.

Figure 2 shows the schematic diagram of the set up overflow-controlled declining rate filtration. Raw water after coagulation and settlement or after coagulation alone (as in direct filtration alone) is directed towards the overflow distributor tank. The overflow level in the distributor tank can be adjusted to provide flexibility in response to changes in the overall rate of filtration or changes in raw water quality and seasonal changes in temperature, etc. Water flows to each filter through feeder pipe fitted with flow restricting orifice as well as an isolating valve. The major turbulent head loss occurs in the flow-restricting orifice whereas the isolating valve is fully opened during normal operation of the filter and hence has negligible head loss. The flow towards each filter varies in accordance with the water level difference between the over flow tank and water level in the individual filters. Accordingly, filter C in Figure 2, which is assumed most recently backwashed, has the lowest water level and hence highest filtration rate. On the other hand, filter $\mathrm{A}$ is the dirtiest filter and has the highest water level and lowest filtration rate. As filtration progresses, the water level in the distributor tank rises as well as the levels in each filter in response to head loss development while maintaining the same overall flow rate through the system.

When water level in the dirtiest filter (filter A) reaches the maximum level, this particular filter is isolated using the isolating valve and backwashed while the remaining filters continue to operate. During this time, the water level in the distributor tank rises as expected as well as the levels in each of the remaining filters in operation.

When filter $A$ is put back in operation after being backwashed, the water levels in all the filters drop down since filter A now takes the highest flow rate as expected. A question may arise as to what is essentially new with this set up that makes it different from the normal declining rate filter. The answer lies in the 


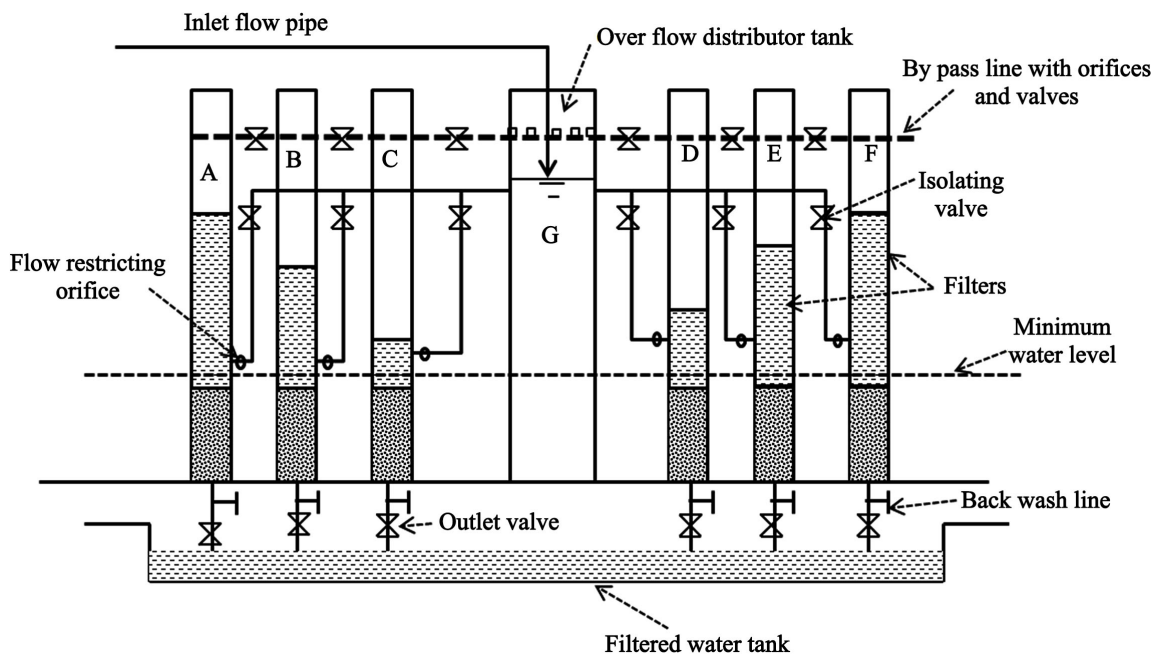

Figure 2. Schematic layout of the overflow controlled declining rate filter model.

provision of separate water levels in each filter, the possibility of varying the overflow water level in the distributor tank and provision of the flow-restricting orifice in the inlet flow pipe to each of the filters. The variation of flow rate in each of the filters is entirely dependent on the water level difference between the individual filters and the distributor tank. This is modeled according to Equation (1):

$$
q_{i}=C_{i} \sqrt{H_{D}-H_{i}}
$$

where

$q_{i}=$ the rate of filtration in filter $i$,

$H_{D}=$ is height of water level in the distributor tank,

$H_{i}=$ the height of water level in the filter $i$ and

$C_{i}=$ orifice head loss parameter corresponding to filter $i$ at a particular stage of filtration.

Figure 3 shows a schematic diagram of the flow rate variation with the water level difference. the distribution of flow rates among the filters can be optimised by studying the changes in flow rates with respect to variables such as the water level in the distributor tank, the orifice head loss and head loss in the filters as filtration progresses. For example, by increasing the water level in the distributor tank, it is possible to increase the proportion of flow going to the dirtiest filter. This can be observed from the differential rate of change of flow rate with respect to change in water level in the distributor tank shown in Equation (2):

$$
\frac{d q_{i}}{d H_{D}}=\frac{C_{i}}{2 \sqrt{H_{D}-H_{i}}}
$$

For example, when increase in coagulant dosage necessitates reducing the ratio of maximum to minimum filtration rates among the filters, this can be achieved by increasing the water level in the over flow tank. This will redistribute the flow from the cleaner filter to the dirtier ones. Conversely, because of 


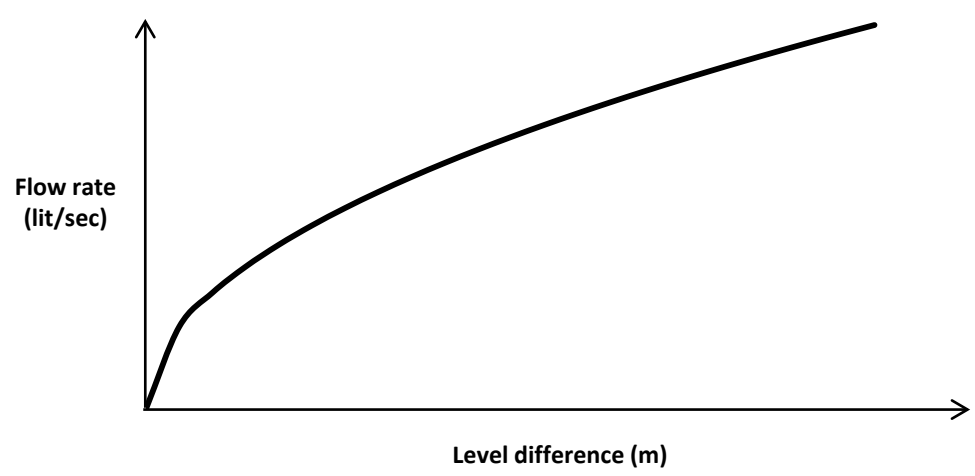

Figure 3. Variationof flow rate with level difference between filter and over flow tank.

increase in plant flow rate in response to increase in demand, the resulting decrease in the ratio of maximum to minimum flow rates can be counteracted by lowering the water level in the distributor tank, an action that will take flow away from the dirtiest filters and redistributes it to the cleaner filters.

In addition, the normal water level of operation in the distributor tank can be fixed in accordance with head loss characteristic of the orifice and laminar flow in the filter media. It is possible to study these characteristics in order to come up with optimal level of water in the distributor tank and optimum orifice size restricting flow in each of the filters.

As is normal with the operation of declining rate filters, the inlet flow distribution in this new filter system does not require special provision for uniform flow distribution, the same advantage achieved in the traditional declining mode of filtration. The settled water is directed towards the overflow tank from which water flow to the different tanks depending on the level difference that exists between the water level in the overflow tank and that of the water level in the individual filters as explained above.

\section{Benefits and Outcomes of the Proposed Design of Overflow Controlled Declining Rate Filtration}

The proposed design concept, which is based on overflow control of declining rate filtration, is expected to resolve some of the operational problems related to declining rate filter performance and add additional flexibility in terms of overall design and routine operation. By providing added control mechanisms in the proposed declining filter set up, it is possible to achieve increased filter performance measured in terms of filter water quality, filter run time, distribution of filtration rate and total filter water throughput. The proposed design, therefore, leads to a declining filter set up that has better efficiency of performance in terms of flow rate distribution, filter run time, filtrate water quality and overall cost. It also means a greater degree of freedom in optimization of the design of filter performance because of the added control variables of the proposed filter setup In addition; factors contributing to sudden changes in flow rate can be 
manipulated in order to control these changes so that the filter's overall performance can be optimized. The result would be an improved filter setup that has stable performance in terms of flow rate changes and filter deposition throughout the cycle of operation of the filter. It also means better control of filter operation and performance because of added flexibility in the filter setup. The proposed design setup offers significant solution alternatives to existing operational problems of declining rate filters, which are often difficult to change or adjust because of the inherent inflexibility present in their setup and operation. The proposed design concept also offers added operational flexibility to short-term as well as long-term changes in design variables. Through this proposed new setup, there will be more flexibility in filter operation and performance and better adaptability to changing conditions during the life of operation of a filtration plant.

The resultant benefit of the proposed filter technology to water treatment plant operating agencies would be stability of filter performance in response to changes in filtration variables, flexibility in adapting filter performance to satisfy desired water quality standard as well as flow rates and filter run lengths. In addition, the overall economy and performance of filters can be improved through greater flow run, reduced filter washing frequency and reduced capital cost of the filter setups required of this technology.

\section{Possibility of Running the Filters in Constant Filter Rate Mode}

Apart from providing increasing flexibility in design and performance, the proposed model through over low control allows the filters to run in constant rate filtration mode. It is, therefore, possible to label the proposed design a dual mode filter enabling operation both in constant rate or declining rate modes. In order to set up the model to operate in constant flow rate mode, the maximum water level in the overflow distributor tank is set to a level higher than the maximum allowable water level rise just before back washing. If the water level in the over flow distributor tank is set to such level higher than the maximum water level rise in the filters, it means that there will be no over flow being thrown out from either of the filters to any one of the other filters. Each individual filter, therefore, operates independently of the other filters. However, the question arises as to how the model can distribute the flow equally from the overflow tank to the filters. A means /device for constant flow distribution among the filters is a requirement of constant rate filters. The answer to the question is made in this model by setting flows to be distributed through the bypass line that is provided with orifice holes in the tank hole as well as valves for isolating each filter (Figure 2). The orifice holes provided through the overflow tank wall provide means for uniform flow distribution among the filters. In this mode of operation, the upstream valves (will be closed) and the orifices that were connected with the declining mode are idle. 


\section{Conclusions}

The proposed design concept of overflow controlled declining rate filtration addresses the bottlenecks of existing declining rate filtration such as lack of flexibility to changes in filter variables with an in-built in self-operating mechanism, flow rate fluctuation and rise of water levels during washing.

Declining rate filters are only provided with downstream orifice as a means of restricting flow rate fluctuation. The lack of flexibility of operation of declining rate filters is evident as when the flow rate changes (due to increased demand, or changes in environmental variables such as temperature for example) the flow distribution among the filters will change. Higher flow rates typically reduce the difference between maximum and minimum rate of flow among the filters. On the other hand reduction of the coagulant dosage will decrease such difference. Such change in flow distribution may be far from the optimum required. Declining rate filters are, therefore, considered optimum in their performance only with respect to narrowly defined operational conditions.

The need for an alternative set up is hereby proposed with the concept of an over flow controlled declining rate filtration with the aim of adaptation of the filter performance to changes in design variables without major structural adjustment. In this proposed setup of over flow controlled declining rate filtration, the water levels in each filter are separate while the whole setup is connected through a common over flow tank. Such proposed set up allows flexibility in individually controlling the flow rate in individual filters. The flow in individual filters would vary according to the level difference between the water level in the over flow tank and the level of water in the individual filters.

Additional flexibility is provided through valve set up and adjusting the maximum level of water in the over flow tank apart from the flow restricting orifice provided downstream. For example, increasing the water level in the over flow distributor tank increases the proportion of flow going to the dirtiest filter. On the other hand a decrease in coagulant dosage can be counteracted by decreasing the water level in the overflow tank there by maintaining a greater difference between the maximum and minimum flow rates among the different filters. Similarly, lowering the water level in the overflow distributor tank assists in counteracting increased water flow rates due to increase water demand which tends to decrease the ration between the maximum and minimum flow rates among the different filters. The proposed setup can also run as constant rate filters by setting the water level in the over flow distributor tank to the maximum and letting the flow pass through the bypass line that is provided with uniform flow distribution orifice holes at the tank walls and valves for isolating each filter.

The overall advantage of this proposed set up to water treatment plants would be stable filter performance in response to changes in filtration parameters, and flexibility in adjusting filter performance to satisfy desired standards of operation such as filter run length, flow rates and water quality. Moreover, economical 
as well as optimal performance can be realized through greater filter run, reduced filter washing frequency and reduced capital cost of the filter setups required of this technology.

\section{Conflicts of Interest}

The authors declare no conflicts of interest regarding the publication of this paper.

\section{References}

[1] Gao, P., Xue, G., Song, X.S. and Liu, Z.H. (2012) Depth Filtration Using Novel Fiber-Ball Filter Media for the Treatment of High-Turbidity Surface Water. Journal of Separation and Purification Technology, 95, 32-38. https://doi.org/10.1016/j.seppur.2012.04.010

[2] Cornwell, D., Macphee, M., Brown, R. and Via, S. (2003) Demonstrating Cryptosporidium Removal Using Spore Monitoring at Lime-Softening Plants. Journal of the American Water Works Association, 95, 124-133. https://doi.org/10.1002/j.1551-8833.2003.tb10367.x

[3] Spellman, F. (2009) Handbook of Water and Wastewater Treatment Plant Operations. 2nd Edition, Taylor \& Francis, Boca Raton, New York. https://doi.org/10.1201/9781420075311

[4] Crittenden, J., Trussell, R.H., Howe, K. and Tchobanoglous, G. (2012) Water Treatment Principles and Design. John Wiley \& Sons, New York. https://doi.org/10.1002/9781118131473

[5] Valentukeviciene, M. (2009) Applying Backwash Water in Order to Enhance Removal of Iron and Ammonium from Spent Filters with Fresh Filter Media. Environment Protection Engineering, 35, 135-144.

[6] Bodzek, M. and Konieczny, K. (2008) Application of Membrane Processes in Water Treatment-State of Art. Instal, 4, 58-61. (In Polish)

[7] American Water Works Association, AWWA (1991) Full-Scale Valuation of Declining and Constant Rate Filtration. American Water Works Association, Denver.

[8] Sabogas, L.P. and DiBernardo, L. (2009) Seleção de tecnologias de tratamento de água. LdiBe, São Paulo.

[9] Libhaber, M. and Jaramillo, A.O. (2012) Sustainable Treatment and Reuse of Municipal Wastewater: For Decision Makers and Practicing Engineers. IWA Publishing, London, 434-441. https://doi.org/10.2166/9781780400631

[10] Akgiray, O. and Saatci, M. (1998) A Critical Look at Declining Rate Filtration Design. Water Science and Technology, 38, 89-96. https://doi.org/10.2166/wst.1998.0240

[11] Mackie, R.I., Dabrowski, W. and Zielina, M. (2003) Numerical Study of a Rational Rule for the Operation of Variable Declining Rate Filters in Response to Changes in Raw Water Quality. Environment Protection Engineering, 29, 45-52.

[12] Dibernardo, L. (1987) Designing Declining-Rate Filters. Filtration and Separation, 338-341.

[13] Dabrwoski, W. (2011) Rational Operation of Variable Declining Rate Filters. Environment Protection Engineering, 37, 35-53.

[14] Cleasby, J.L. and Dibernardo, L. (1980) Declining Rate versus Constant Rate Filtration. Journal of Environmental Engineering Division of the American Society of 
Civil Engineers, 106, 1023-1043.

[15] Dabrwoski, W. (2006) The Progression of Flow Rates in Variable Declining Rate Filter Systems. Acta Hydrochimica et Hydrobiológica, 34, 442-452. https://doi.org/10.1002/aheh.200500647

[16] Cleasby, J.L. (1993) Status of Declining Rate Filtration Design. Water Science and Technology, 27, 151-164. https://doi.org/10.2166/wst.1993.0224

[17] Escobar, J. (1992) Influência da capacidade de armazenamento de água a montante dos filtros nofuncionamento de sistemas de filtração com taxa declinante. Dissertação para Obtenção dotitulo de Mestre em Hidráulica e Saneamento, Escola de Engenharia de São Carlos, Universidade de São Paulo, Brasil.

[18] Perera, T.L., Torres, L.P. and Cruz, V.C. (2014) Influence of the Flow Control (Declining Rate-Constant Rate) on the Filtration Process in Drinking Water Treatment. Ingeniería y Universidad, 18, 217-234.

https://doi.org/10.11144/Javeriana.IYU18-2.ifcd

[19] Mackie, R.I., Zielina, M. And Dabrowski, W. (2003) Filtrate Quality from Different Filter Operations. Acta Hydrochimica et Hydrobiologica, 31, 25-35. https://doi.org/10.1002/aheh.200390012

[20] Marzec, J. and Dabrowski, W. (1996) Operation of Rapid sand Filters under Fluctuable Water Turbidity. Ochrona, Srodowiska, 2, 19-21. (In Polish)

[21] Dabrwoski, W. and Mackie, R. (1997) Dynamics of Variable Declining Rate Filters during Backwash. Archives of Hydro-Engineering and Environmental Mechanics, 44, 69-83.

[22] Kawamura, S. (2000) Integrated Design of Water Treatment Facilities. 2nd Edition, John Wiley \& Sons, New York.

[23] Ziellina, M. and Dabrowski, W. (2011) Impact of Raw Water Quality on Operation of Variable Declining Rate Filter Plants. Environment Protection Engineering, 37, 133-140.

[24] Baylis, J.R. (1959) Nature and Effects of Filter Backwashing. Journal of the American Water Works Association, 51, 126-137. https://doi.org/10.1002/j.1551-8833.1959.tb21130.x

[25] Hilmoe, D.J. and Cleasby, J.L. (1986) Comparing Constant-Rate and Declining-Rate Direct Filtration of a Surface Water. Journal of the American Water Works Association, 78, 26-34. https://doi.org/10.1002/j.1551-8833.1986.tb02762.x

[26] Degremont, S. (2016) Declining Rate Filtration. https://www.suezwaterhandbook.com/processes-and-technologies/filters/filter-cont rol-and-regulation/declining-rate-filters

[27] Libhaber, M. and Jaramil, A.O. (2012) Sustainable Treatment and Reuse of Municipal Wastewater. IWA Publishing, London.

[28] Murat, E.O., Akgirayb, M. and Ebubekir, Y. (2013) An Experimental Investigation on the Hydraulic Behavior of Declining Rate Filtration. Desalination and Water Treatment, 51, 6137-6147. https://doi.org/10.1080/19443994.2013.763736

[29] Boller, M. and Kavanaugh, M. (1995) Particle Characteristics and Head Loss Increase in Granular Media Filtration. Water Research, 29, 1139-1149. https://doi.org/10.1016/0043-1354(94)00256-7 